\title{
LES LARVES DE BRACHYCENTRIDAE (TRICHOPTERA) DE LA FAUNE DE FRANCE. TAXONOMIE ET ECOLOGIE
}

\author{
par H. DÉcamps
}

SOMMAIRE

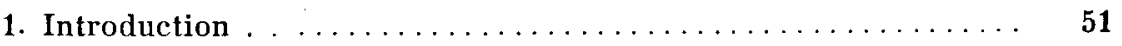

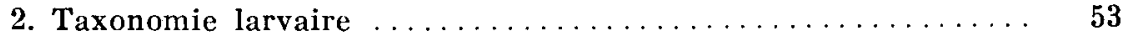

2.1. Caractères généraux de la famille ........... 53

2.2. Caractères utilisés dans la séparation des espèces ... 54

2.3. Description des espèces $\ldots \ldots \ldots \ldots \ldots \ldots \ldots \ldots \ldots .55$

3. Notes écologiques $\ldots \ldots \ldots \ldots \ldots \ldots \ldots \ldots \ldots \ldots \ldots \ldots \ldots \ldots .59$

3.1. Répartition écologique $\ldots \ldots \ldots \ldots \ldots \ldots \ldots \ldots \ldots \ldots \quad 59$

3.2. Répartition géographique $\ldots \ldots \ldots \ldots \ldots \ldots \ldots \ldots \ldots, 60$

3.3. Captures en France .................... 60

4. Clé de détermination des espèces françaises $\ldots \ldots \ldots \ldots \ldots 68$

5. The french Brachycentridae larvae - Key ............. 69

6. Die französischen Brachycentriden Larven. - Bestimmungs-

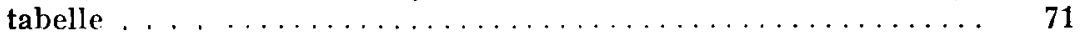

\section{1. - INTRODUCTION}

Les Trichoptères Brachycentridae forment un élément important du benthos des eaux courantes par la densité de leurs populations larvaires, notamment sur les pierres et les mousses du rhithron. En Europe, cette famille se compose de trois genres comprenant une vingtaine d'espèces. Douze ont été signalées en France :

Brachycentrus montanus Klapalek, 1892,

Brachycentrus subnubilus Curtis, 1834, Oligoplectrum maculatum (Fourcroy, 1785), Micrasema cinereum Mosely, 1930, 
Micrasema difficile Mosely, 1934',

Micrasema longulum MacLachlan, 1876

Micrasema minimum MacLachlan, 1876,

Micrasema moestum (Hagen, 1868),

Micrasema morosum (MacLachlan, 1868),

Micrasema setiferum (Pictet, 1834),

Micrasema togatum (Hagen, 1863),

Micrasema tristellum MacLachlan, 1876.

Micrasema setiferum, citée une seule fois des Vosges d'après un exemplaire femelle [Mc LAcHLAN 1884] $]_{\curlyvee}$ doit être considérée comme possible mais douteuse pour la faune de France.

Deux espèces sont bien connues à l'état larvaire - Brachycentrus subnubilus et Oligoplectrum maculatum - grâce aux descriptions de Hickin (1943) et LepNeva (1966) pour la première, de Nielsen (1943) pour la seconde. Cinq espèces ne sont connues que par des travaux datant du début du siècle : Brachycentrus montanus, Micrasema longulum, $M$. minimum, $M$. setiferum et $M$. cinereum $^{2}$. Enfin, les stades aquatiques de cinq espèces sont encore inconnus : Micrasema togatum, $M$. difficile, $M$. moestum, $M$. morosum et $M$. tristellum.

Cette note a pour objet :

- de préciser la distribution et la taxonomie larvaire de ces espèces;

- de permettre l'identification directe des stades aquatiques des Brachycentridae de la faune de France.

Plusieurs collègues m'ont envoyé du matériel de leur propre collection, J. Baudoin (Clermont-Ferrand), L. Botosaneanu (Bucarest), M. Bournaud (Lyon), W. Döhler (Klingenberg/Main), J. Giudicelli (Marseille). M. Thibault (Biarritz) et J. Verneaux (Besançon). J. M. Elliotr (Ambleside) a relu et corrigé la clé de détermination en anglais.

Je les prie d'accepter mes vifs remerciements.

1. Un examen du type de Micrasema vestitum Navas nous a montré que Micrasema difficile Mosely et Micrasema vestitum Navas étaient deux espèces distinctes [Botosaneanu et Décamps en préparation]. La synonymie adoptée précédemment [DÉcamps 1967] doit donc être abandonnée; l'espèce récoltée sur le versant nord-pyrénéen par Mosely [1934] et Décamps [1967 a, b, 1968] doit s'appeler Micrasema difficile.

2. La description publiée par Klapalek [1917] pour Micrasema togatum se rapporte en fait à Micrasema cinereum [GIUdicellı 1968]. 


\section{TAXONOMIE LARVAIRE}

\subsection{Caractères généraux de la famille.}

Les larves de Brachycentridae ont en commun :

- un mésonotum entièrement chitineux,

- l'absence de corne prosternale,

- l'absence de mamelons sur le $1^{\text {er }}$ segment abdominal.

Ces caractères les distinguent des autres familles européennes à larves éruciformes. Les larves de Limnephilidae, Goeridae et Lepidostomatidae sont pourvues de cornes prosternales. Les autres familles voisines, sans cornes prosternales, possèdent des mamelons sur le $1^{\text {er }}$ segment abdominal.

La figure 1 indique le schéma général d'une larve de Brachycentridae et la nomenclature utilisée.

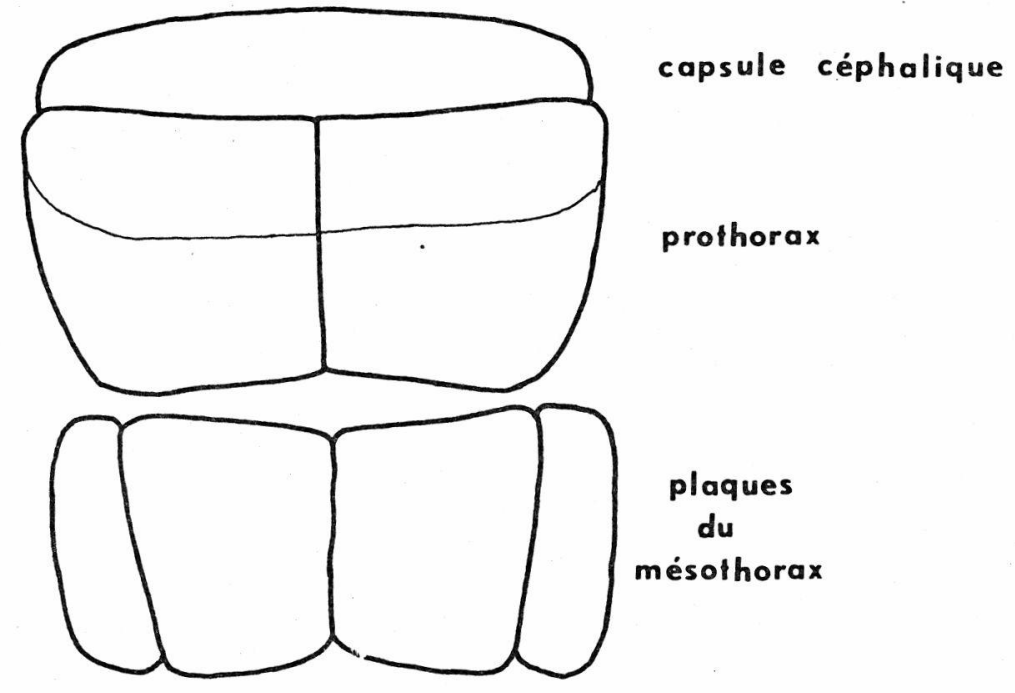

Fig. 1. - Schéma général. 


\subsection{Caractères utilisés dans la séparation des espèces.}

Etui larvaire (fig. 2 à 12). - Les étuis larvaires des Brachycentridae sont variés et, pour une espèce donnée, assez constants pour fournir de bons caractères de détermination.

L'étui d'Oligoplectrum maculatum (fig. 7) est composé de particules minérales, sa section est arrondie. Il est droit, effilé vers l'arrière, parfois très long. Les Brachycentrus ont des étuis quadrangulaires, composés de fragments végétaux disposés perpendiculairement à l'axe. Les arêtes sont généralement plus vives chez B. montanus que chez B. subnubilus. Pour les Micrasema, on peut distinguer :

— les étuis de nature minérale : $M$. moestum et $M$. minimum;

- les étuis de nature végétale : $M$. tristellum et $M$. togatum;

- les étuis entièrement secrétés : droits chez $M$. morosum, arqués vers l'arrière chez $M$. longulum et $M$. cinereum;

- les étuis mixtes avec des parties minérales et des parties secrétées : $M$. difficile.

La membrane fermant l'extrémité postérieure de l'étui présente une ouverture circulaire chez $M$. minimum ( $f i g .27$ ); elle est munie de pointes radiales chez les autres Micrasema : 3 chez $M$. longulum (fig. 39) 3 à 4 chez $M$. moestum, 4 à 5 chez $M$. difficile, $M$. morosum (fig. 32), M. tristellum (fig. 43), M. cinereum et 5 ou 6 chez M. togatum.

Capsule céphalique. - Les colorations sont dans l'ensemble uniformes, sauf pour Brachycentrus subnubilus (fig. 18). La forme de la capsule et son aplatissement plus ou moins grand sont caractéristiques de certaines espèces : $M$. difficile, très aplatie; $M$. tristellum, arrondie. Les figures en vue dorsale indiquent la présence possible de protubérances au niveau des yeux (M. longulum) ou des différences dans les formes des bords latéraux.

Plaques du mésothorax. - Le mésothorax permet une bonne distinction des espèces. Il comprend 4 plaques chitineuses chez Oligoplectrum, les Brachycentrus et certaines Micrasema : $M$. minimum, M. setiferum, $M$. moestum et $M$. morosum. On ne compte plus que 2 plaques chitineuses chez les autres Micrasema : $M$. longulum, $M$. cinereum, $M$. difficile, $M$. tristellum et $M$. togatum. Wiggins [1965] a noté la présence de 4 plaques mésothoraciques chez une seule des Micrasema nord-américaines actuellement connues à l'état larvaire. Ce caractère est peut-être plus répandu chez les Micrasema européennes puisque pour la France, 4 espèces sur 9 possèdent 4 plaques mésothoraciques.

Patres. - L'examen des pattes méso et métathoraciques permet de distinguer les 2 genres Oligoplectrum et Brachycentrus du genre Micrasema. Les caractères utilisés sont : (1) la longueur relative du 
fémur et du tibia, (2) la présence ou l'absence d'un processus ventral aux parties distales des tibias. Des saillies spiniformes peuvent être observées sur les hanches des pattes méso et métathoraciques d'Oligoplectrum maculatum et de Brachycentrus subnubilus.

\subsection{Description des espèces.}

Nous donnons ici les principales références, le matériel examiné et les dimensions au dernier stade pour toutes les espèces. Une courte description est ajoutée pour les larves peu connues ou inconnues.

Oligoplectrum maculatum. - La morphologie de la larve a été étudiée en détail par NiELSEN [1943].

Matériel examiné : une dizaine de larves récoltées dans la rivière le Lez à Moulis en Ariège (leg. Bournaud) .

Dimensions au dernier stade :

- longueur maximale de l'étui : $18 \mathrm{~mm}$;

- longueur maximale de la larve : $9 \mathrm{~mm}$;

- largeur de la capsule céphalique : 0,6-0,7 $\mathrm{mm}$.

Brachycentrus subnubilus. - La larve est bien connue grâce aux descriptions et dessins de Hickin [1943] et de LepNeva [1966].

Matériel examiné : une dizaine de larves provenant de la région lyonnaise (leg. Bournaud), de la Loue, affluent du Doubs (leg. Verneaux) et de la vallée de l'Ost à Turnu Rosu en Roumanie (leg. Botosaneanu).

Dimensions au dernier stade :

— Iongueur maximale de l'étui : $13 \mathrm{~mm}$;

- longueur maximale de la larve : $12 \mathrm{~mm}$;

- largeur de la capsule céphalique : 0,9-1,1 mm.

La coloration est jaune-clair avec des plages brunes plus foncées.

Brachycentrus montanus. - La larve est connue par des descriptions anciennes : avec Lestage [1921], on peut citer KLAPALEK [1893] et Ulmer [1903].

Matériel examiné : une vingtaine de larves provenant d'Auvergne (leg. BaudorN) et des Carpates méridionales : vallée du Jin (leg. Botosaneanu).

Dimensions au dernier stade :

- longueur maximale de l'étui : $11 \mathrm{~mm}$;

- longueur maximale de la larve : $8 \mathrm{~mm}$;

- largeur de la capsule céphalique : $0,9 \mathrm{~mm}$.

La coloration est uniformément marron-foncé. 
La bande longitudinale noire de la bordure dorsale du fémur, si nette chez B. subnubilus, est moins visible chez B. montanus. Elle existe cependant chez les deux espèces mais apparaît plus nettement sur le jaune-clair de la coloration d'ensemble de $B$. subnubilus que sur le marron-foncé de $B$. montanus. Ce caractère n'a donc pas été inclus dans la clé de détermination.

Micrasema minimum. - La larve a été décrite par Ulmer [1903, 1909] et par Thienemann [1905].

Matériel examiné : nombreuses larves récoltées en vallée d'Aure, dans les Pyrénées centrales.

Dimensions au dernier stade :

- longueur maximale de l'étui : $10 \mathrm{~mm}$;

- longueur maximale de la larve : $8 \mathrm{~mm}$;

- largeur de la capsule céphalique : 0,6-0,7 mm.

L'étui est composé de fines particules minérales séparées par un ciment secrété. Les caractères principaux de cette larve sont :

- la présence de 4 plaques mésothoraciques;

- l'aplatissement de la capsule céphalique;

- la coloration brun-foncé des parties chitinisées;

- la présence d'une ouverture circulaire sur la membrane de fermeture postérieure de l'étui.

Micrasema moestum. - La larve n'avait pas encore été décrite.

Matériel examiné : nombreuses larves récoltées dans la rivière le Volp en Ariège.

Dimensions au dernier stade :

- longueur maximale de l'étui : $11 \mathrm{~mm}$;

- longueur maximale de la larve : $8 \mathrm{~mm}$;

- largeur de la capsule cépalique : 0,7-0,9 $\mathrm{mm}$.

Etui formé de particules minérales accolées, sans ciment visible (fig. 5). Ouverture postérieure limitée par 3 à 5 pointes radiales comme l'espèce suivante Micrasema morosum (fig. 32). Coloration marron, s'éclaircissant vers l'arrière, sur le mésonotum notamment. Capsule céphalique à bords latéraux arrondis (fig. 29). Partie dorsale légèrement arrondie en vue latérale (fig. 30 ). Taches d'insertions musculaires généralement bien délimitées. Mésothorax avec 4 plaques chitinisées. Bord latéral des plaques centrales oblique et rectiligne (fig. 28).

Micrasema morosum. - La larve n'avait pas encore été décrite.

Matériel examiné : nombreuses larves récoltées dans des sources et ruisseaux des Pyrénées centrales.

Dimensions au dernier stade :

- longueur maximale de l'étui : $13 \mathrm{~mm}$; 
- longueur maximale de la larve : $11 \mathrm{~mm}$;

- largeur de la capsule céphalique : 0,9-1 mm.

Etui entièrement secrété, droit. Coloration marron, partie postérieure du pronotum légèrement plus claire. Capsule céphalique à bords droits, convergeant vers l'arrière à partir des yeux (fig. 31). Partie dorsale légèrement arrondie en vue latérale (fig. 33). Mésothorax aveo 4 plaques chitinisées, le bord latéral des plaques centrales sinueux (fig. 34).

Micrasema longulum. - Des descriptions ont été données par Ulmer [1903, 1909], Thienemann [1905] et Lestage [1921].

Matériel examiné : nombreuses larves récoltées dans les cours d'eau des Pyrénées centrales.

Dimensions au dernier stade :

- longueur maximale de l'étui : $13 \mathrm{~mm}$;

- longueur maximale de la larve : $10 \mathrm{~mm}$;

- largeur de la capsule céphalique : $0,7 \mathrm{~mm}$.

Étui entièrement secrété, arqué vers l'arrière (fig. 11). Ouverture postérieure limitée par 3 pointes radiales. Parties chitinisées chagrinées, de couleur marron. Capsule céphalique avec des protubérances au niveau des yeux (fig. 36). Insertions musculaires visibles. Mésothorax avec deux plaques chitinisées, à angles latéro-antérieurs se prolongeant en arrondi vers l'avant (fig. 38). Tarses des pattes méso- et métathoraciques munis de forts tubercules coniques à la partie distale du bord ventral (fig. 40).

Micrasema difficile. - La larve n'avait pas encore été décrite.

Matériel examiné : nombreuses larves récoltées dans les sources et ruisseaux des Pyrénées.

Dimensions au dernier stade :

- longueur maximale de l'étui : $10 \mathrm{~mm}$;

- longueur maximale de la larve : $7 \mathrm{~mm}$;

- largeur de la capsule céphalique : $0,7 \mathrm{~mm}$.

Etui mixte, en partie minéral, en partie secrété. Parties chitinisées brunes. La capsule céphalique, très aplatie, paraît concave en vue latérale (fig. 48). Elle est presque entièrement cachée par le pronotum en vue dorsale ( $f g .49$ ). Pronotum trapézoïdal, élargi vers l'avant. Mésonotum avec 2 plaques chitinisées d'aspect quadrangulaire.

Micrasema tristellum. - La larve n'avait pas encore été décrite.

Matériel examiné : 3 larves récoltées en Engadine (leg. Nadig). Ces exemplaires m’ont été envoyés par W. DöHLER.

Dimensions au dernier stade:

- longueur maximale de l'étui : $11 \mathrm{~mm}$; 
- longueur maximale de la larve : $7 \mathrm{~mm}$;

- largeur de la capsule céphalique : 0,8-0,9 mm.

Étui formé de fragments végétaux disposés perpendiculairement à l'axe (fig. 3). Ouverture postérieure limitée par 4 ou 5 pointes radiales (fig. 43). Capsule céphalique et pronotum marron foncé. Une aire plus claire, en $\mathrm{Y}$, recouvre la ligne de suture des pleures et de la partie postérieure du frontoclypeus. Mésonotum jaune. Capsule céphalique plus globuleuse que celle des autres espèces du genre, nettement bombée en vue latérale (fig. 46). Yeux sur des protubérances (fig. 45).

Micrasema cinereum. - Larve décrite par KLAPALEK [1917] sous le nom de M. togatum [Giudicelli 1968].

Matériel examiné : une dizaine de larves provenant de la vallée de la Restonica en Corse (leg. Giudrcelli).

Dimensions au dernier stade :

- Iongueur maximale de l'étui : $14 \mathrm{~mm}$;

- longueur maximale de la larve : $10 \mathrm{~mm}$;

- largeur de la capsule céphalique : 0,7-0,9 mm.

Comme chez $M$. longulum, l'espèce continentale à laquelle elle ressemble le plus, la larve de $M$. cinereum possède un étui secréié (fig. 10), légèrement arqué vers l'arrière. Elle présente aussi, aux tarses des pattes méso- et métathoraciques, des tubercules (fig. 41) rappelant les tubercules plus importants de $M$. longulum. Cependant, l'espèce corse diffère de l'espèce continentale par l'absence de protubérance au niveau des yeux (fig. 51) et par l'absence de prolongement aux angles antérieurs du pronotum (fig. 50). La coloration est uniformément brune, légèrement moins foncée sur le mésonotum.

Micrasema togatum. - La larve n'avait pas encore été décrite.

Matériel examiné : 5 larves provenant de la vallée de la Restonica en Corse (leg. Giudicelli).

Dimensions au dernier stade :

- longueur maximale de l'étui : $9 \mathrm{~mm}$;

- longueur maximale de la larve : $8,5 \mathrm{~mm}$;

- largeur de la capsule céphalique : $0,9 \mathrm{~mm}$.

L'espèce continentale voisine est ici $M$. tristellum. Les deux espèces ont en commun un étui composé de fragments végétaux (fig. 3 et 4) et la coloration des parties chitinisées : plus foncée pour la tête et le pronotum chez $M$. tristellum, plus foncée pour la tête et la partie antérieure du pronotum chez $M$. togatum (fig. 54). L'espèce corse diffère de l'espèce continentale par une capsule céphalique à bord dorsal légèrement arrondi et non bombé ainsi que par des protubérances oculaires moins nettes (fig. 53). 
Distinction des deux espèces corses. - Elles diffèrent en premier lieu par la nature de l'étui, secrété chez $M$. cinerum, végétal chez $M$. togatum. La coloration est aussi caractéristique : uniformément brun foncé chez $M$. cinereum, elle est plus claire chez $M$. togatum avec la tête et la partie antérieure du pronotum marron, la partie postérieure du pronotum et le mésonotum jaune.

Autres caractères distinctifs : (1) Tubercules coniques des tarses des pattes méso- et métathoraciques présents chez $M$. cinereum, absents chez $M$. togatum. (2) Capsule céphalique plus arrondie en vue latérale chez $M$. togatum. (3) Plaques chitinisées du mésonotum quadrangulaires chez $\boldsymbol{M}$. cinereum (rappelant $\boldsymbol{M}$. difficile), rectangulaires et allongées transversalement chez $M$. togatum. (4) La pilosité est, dans l'ensemble, plus développée chez $M$. togatum.

\section{3. - NOTES ECOLOGIQUES}

\subsection{Répartition écologique.}

A l'exception de Brachycentrus subnubilus, caractéristique du potamon, les espèces de la famille des Brachycentridae font partie du rhithron [BotosanEANU 1967].

Dans les Pyrénées, la distribution écologique des Brachycentridae peut être résumée ainsi [DÉCAMPs 1968] :

M. morosum : sources et ruisseaux de haute montagne, moins abondante au-dessous de 1000 mètres;

$M$. difficile : sources et ruisseaux de moyenne montagne;

$\boldsymbol{M}$. longulum et $\boldsymbol{M}$. minimum : ruisseaux et petites rivières de basse et moyenne montagne;

$M$. moestum : ruisseaux et petites rivières de plaine;

$O$. maculatum et $B$. subnubilus : rivières de plaine.

En Corse [Givdicelli 1968], M. togatum, vivant dans les sources et petits ruisseaux, peut être considérée comme l'équivalent de $\boldsymbol{M}$. difficile. $\boldsymbol{M}$. cinereum occupe une position semblable à celles de $M$. longulum et de $M$. minimum.

Dans les autres régions, $M$. tristellum a été récoltée dans des sources ou dans leur voisinage, $B$. montanus et $M$. setiferum dans de petits cours d'eau. $O$. maculatum est présente aussi dans de petits cours d'eau hors des Pyrénées.

Enfin, certaines espèces de Micrasema sont plus nettement muscicoles. Ce sont $M$. morosum, $M$. difficile, $M$. longulum et $M$. minimum dans les Pyrénées, $M$. togatum en Corse. 


\subsection{Rẻpartition géographique.}

Les Brachycentridae sont dans leur majorité des espèces montagnardes : en Europe, 7 espèces vivent dans les Pyrénées 8 ont été signalées dans les Alpes, 7 dans le Massif Central et les Mittelgebirge [Botosaneanu 1967]. Pour les Brachycentridae français, on peut distinguer :

- des espèces à distribution européenne. Dans ce groupe, Brachycentrus subnubilus présente la distribution la plus étendue; c'est la seule espèce de la famille présente dans les Iles Britanniques. Moins largement répandues, Oligoplectrum maculatum, Micrasema minimum et $M$. setiferum ont aussi été capturées en diverses régions d'Europe.

- des espèces localisées aux massifs montagneux et leur bordure : Brachycentrus montanus, Micrasema longulum, $M$. moestum, $M$. morosum et $M$. tristellum.

- des espèces endémiques : Micrasema cinereum et $M$. togatum en Corse, Micrasema difficile dans les Pyrénées.

\subsection{Captures en France.}

Les travaux de Berland et Mosely [1936], Maillet et Carasso [1955], Décamps [1967], Baudorn [1968] et Giudicelli [1968] permettent de résumer les captures de Brachycentridae en France.

Par rapport au catalogue des Trichoptères de France [Berland et Mosely 1936], les seules mentions apportant d'importantes nouveautés concernent Brachycentrus montanus et Brachycentrus subnubilus. La première a été signalée en Dordogne [MaIllet et Capasso 1955] (rectification manuscrite des auteurs) et en BasseAuvergne [Baudoin 1968]. La seconde a été signalée en HauteGaronne [DÉcAMPS 1967 a]. 

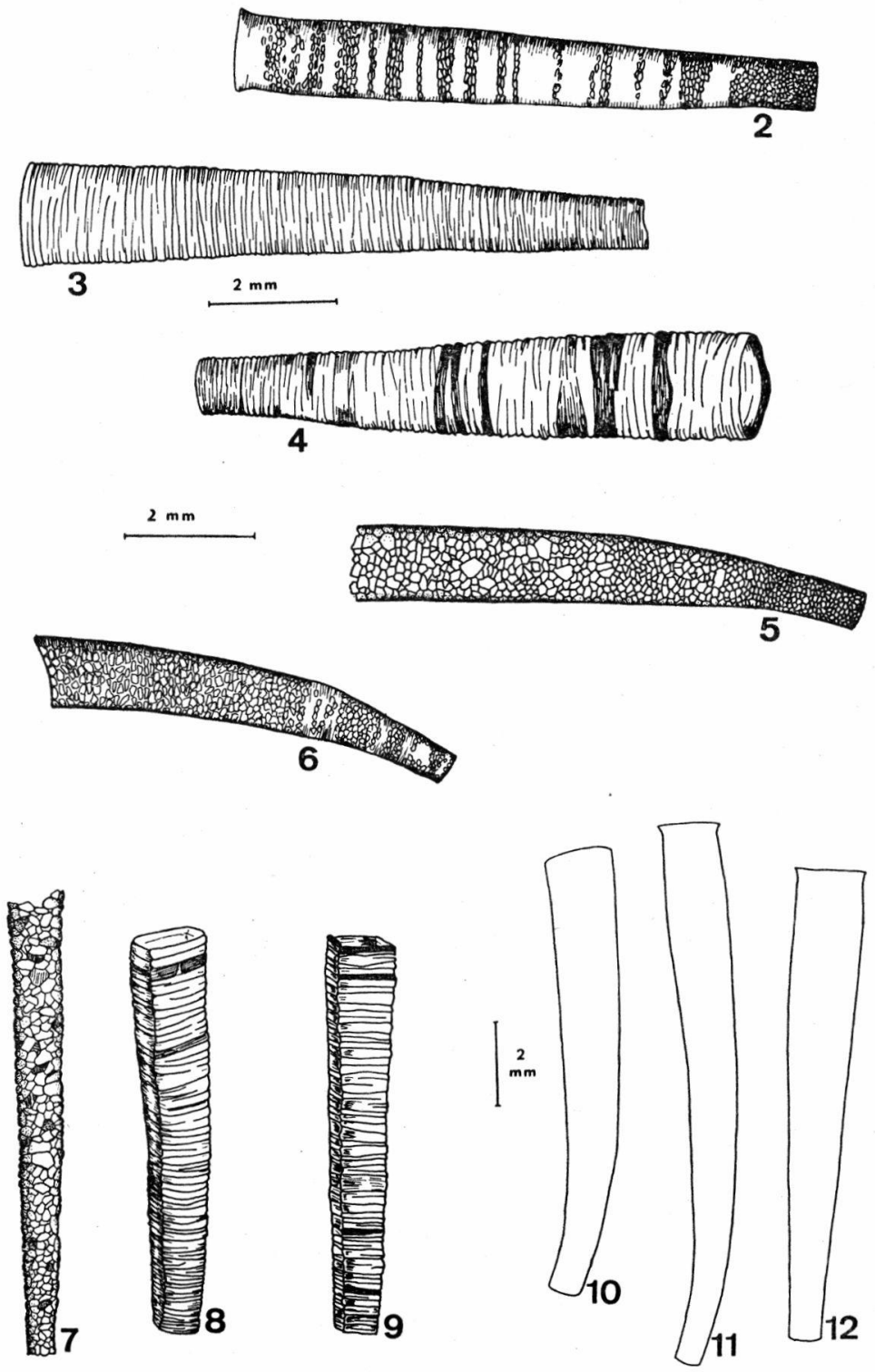

FIg. 2 à 12. - Etuis larvaires de Brachycentridae. 2 : Micrasema difficile. 3 : Micrasema tristellum. 4 : Micrasema togatum. 5 : Micrasema moestum. 6 : Micrasema minimum. 7 : Oligoplectrum maculatum. 8 : Brachycentrus subnubilus. 9 : Brachycentrus montanus. 10 : Micrasema cinereum. 11 : Micrasema longulum. 12 : Micrasema morosum. 


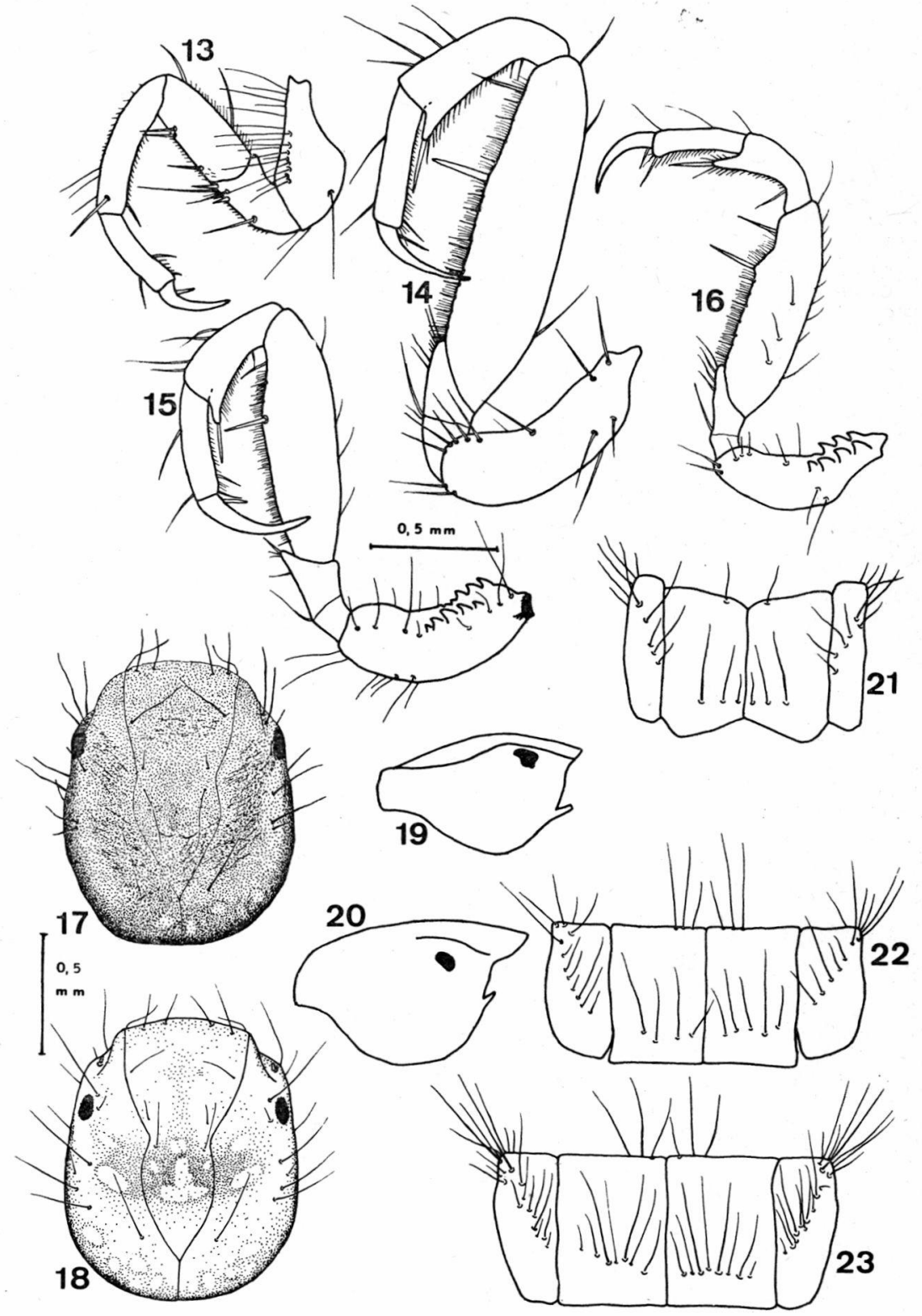

FIg. 13 à 16. - Pattes antérieures) de larves de Brachycentridae. 13 : Micrasema morosum. 14 : Brachycentrus montanus. 15 : Brachycentrus subnubilus. 16 : Oligoplectrum maculatum.

Fig. 17 et 18. - Capsules céphaliques (en vue dorsale) de larves de Brachycentrus. 17 : B. montanus. 18 : B. subnubilus.

Fig. 19 et 20. - Capsules céphaliques vues de profil. 19 : Oligoplectrum maculatum. 20 : Brachycentrus subnubilus.

FIG. 21 à 23. - Plaques mésonotales. 21 : Oligoplectrum maculatum. 22 : Brachycentrus montanus. 23 : Brachycentrus subnubilus. 


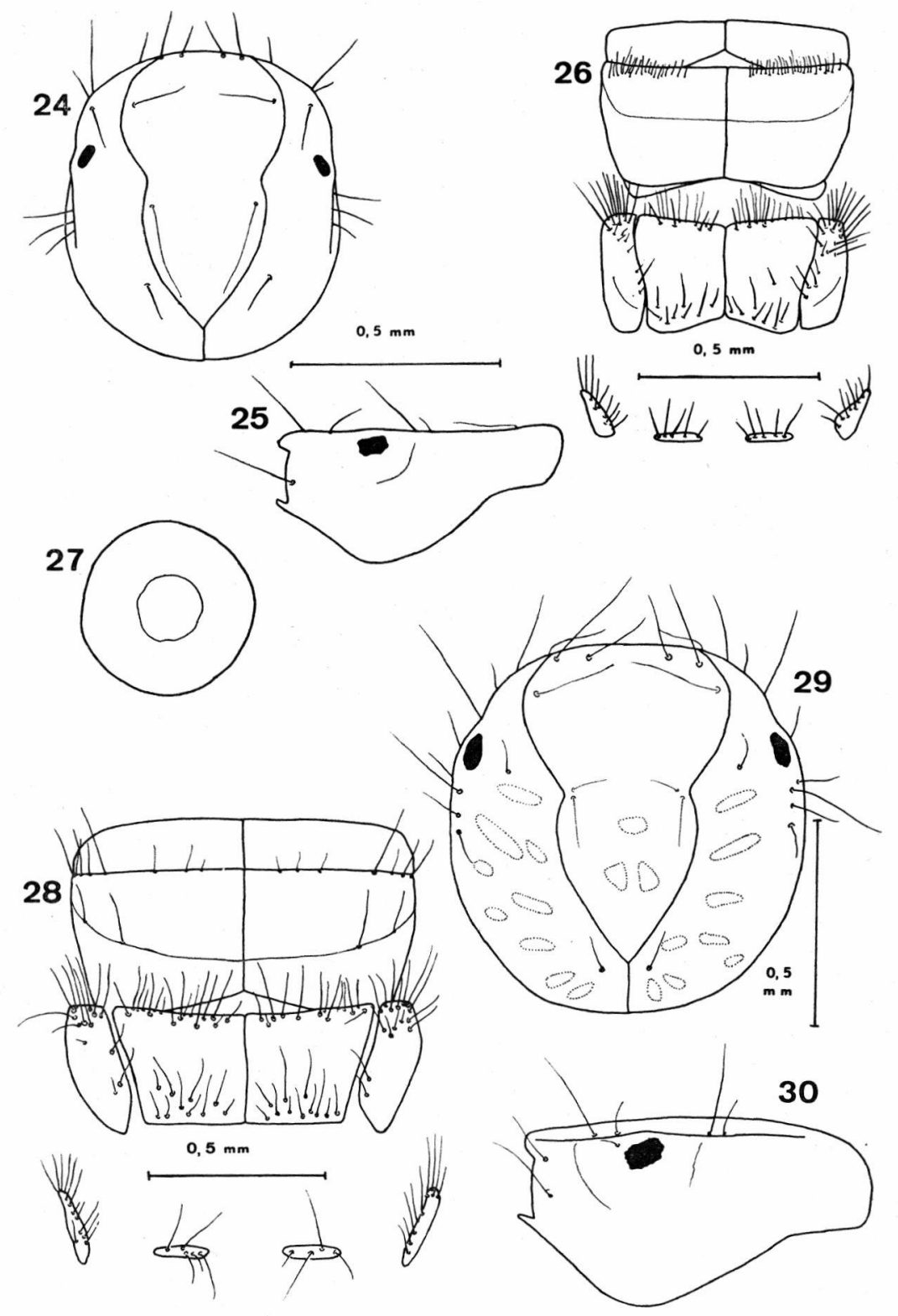

FIg. 24 à 27. - Larve de Micrasema minimum. 24 : capsule céphalique en vue dorsale. 25 : capsule céphalique vue de profil. 26 : tête et plaques thoraciques. 27 : ouverture postérieure de l'étui.

FIG. 28 à 30. - Larve de Micrasema moestum. 28 : tête et plaques thoraciques. 29 : capsule céphalique en vue dorsale. 30 : capsule céphalique vue de profil. 


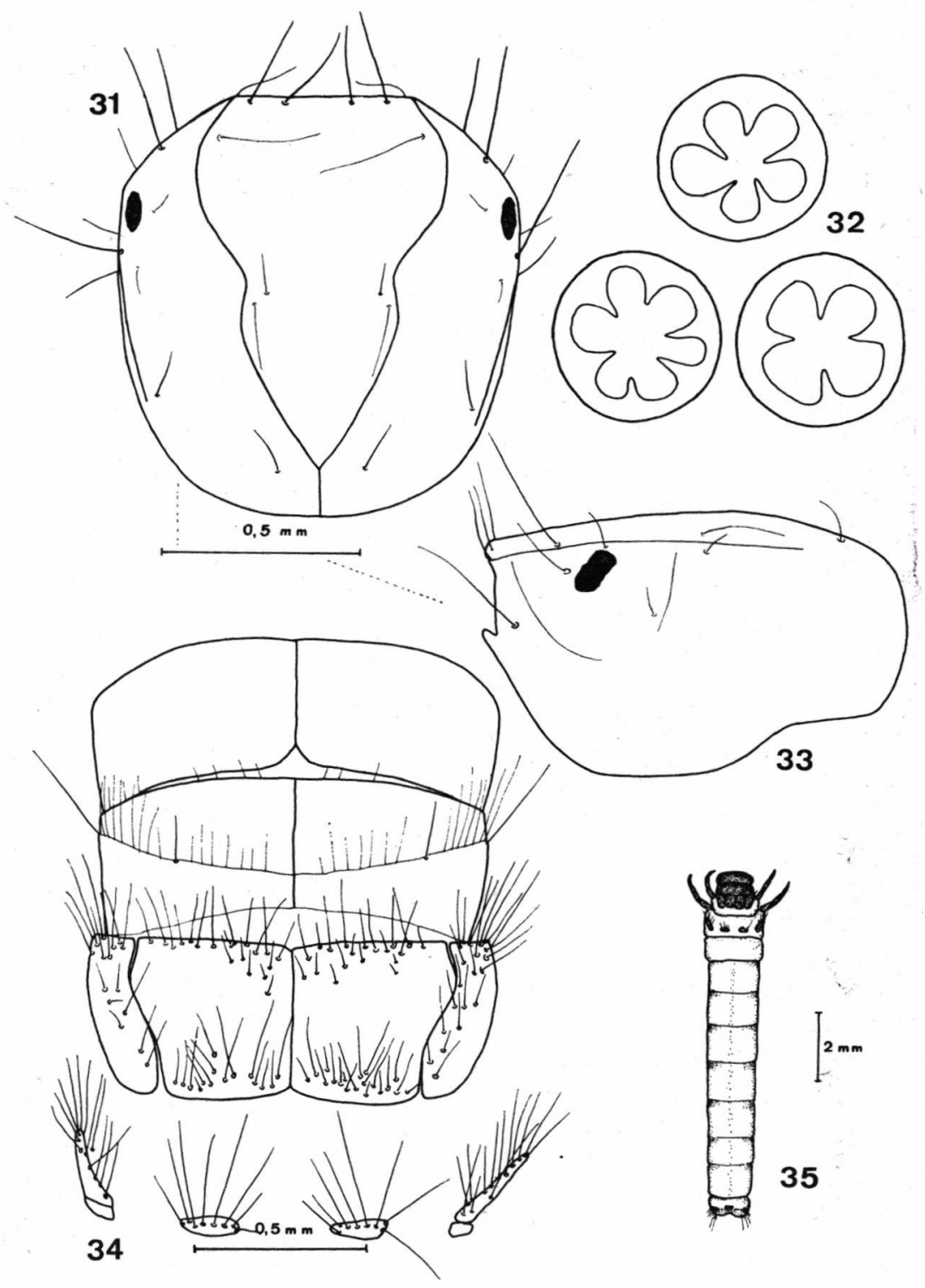

Fig. 31 à 35 . - Larve de Micrasema morosum. 31 : capsule céphalique en vue dorsale. 32 : ouvertures postérieures de l'étui. 33 : capsule céphalique vue de profil. 34 : tête et plaques thoraciques. 35 : habitus. 

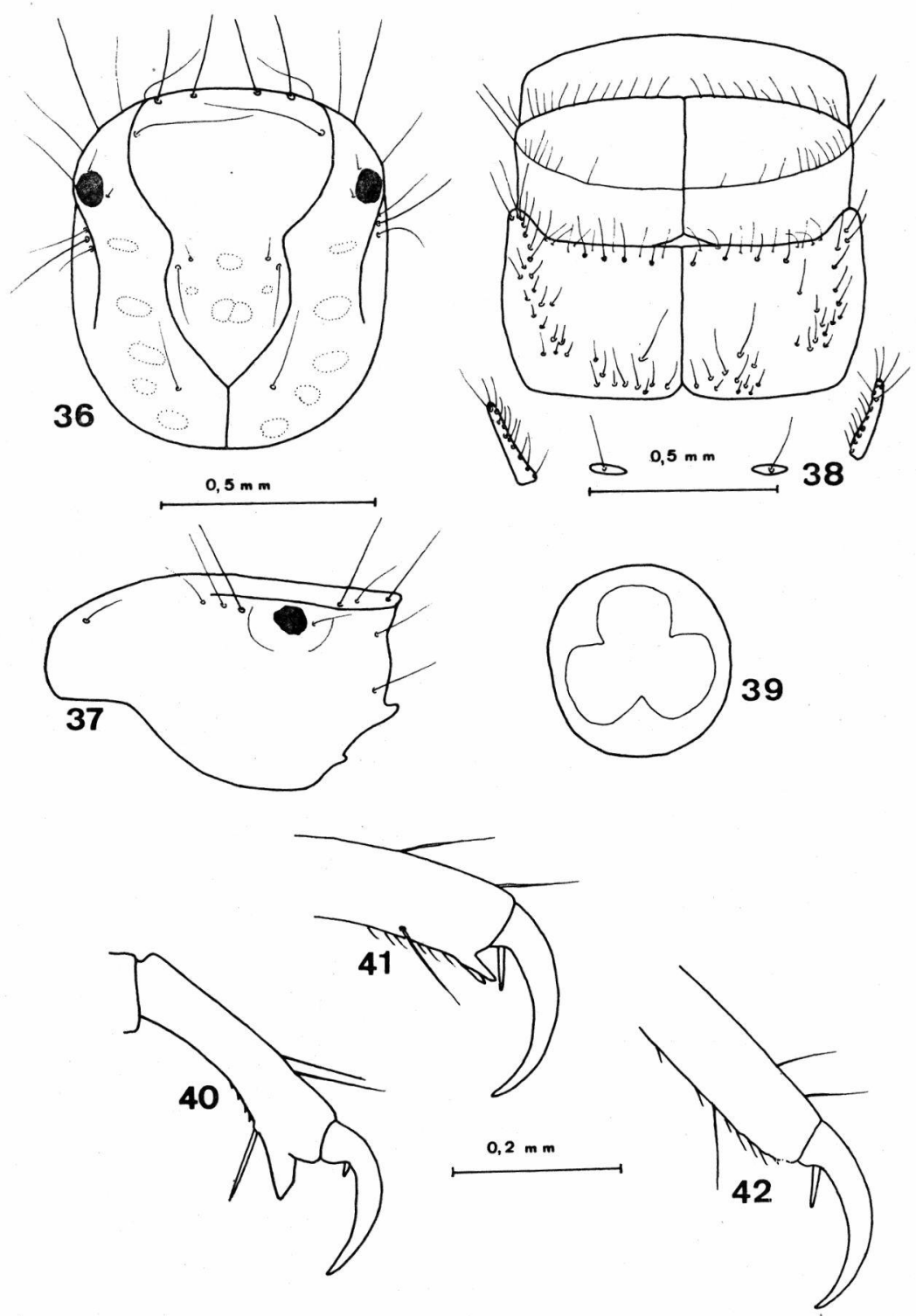

Fig. 36 à 39. - Larve de Micrasema longulum. 36 : capsule céphalique en vue dorsale. 37 : capsule céphalique vue de profil. 38 : tête et plaques thoraciques. 39 : ouverture postérieure de l'étui.

FIG. 40 à 42 . - Tarses des pattes médianes de Micrasema. 40 : M. longulum. $41:$ M. cinereum. $42: M$. togatum. 


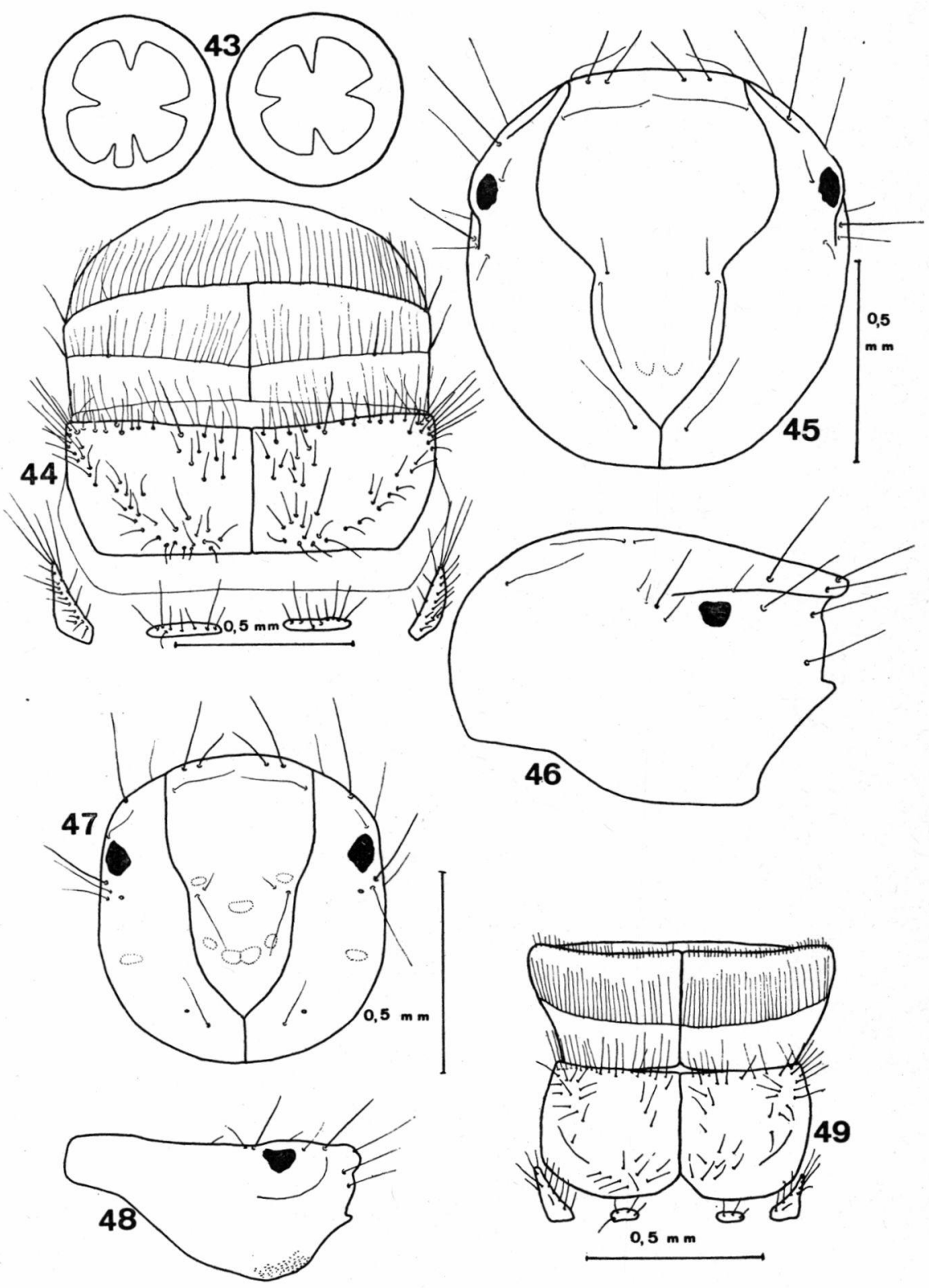

Fig. 43 à 46. - Larve de Micrasema tristellum. 43 : ouverture postérieure de l'étui. 44 : tête et plaques thoraciques. 45 : capsule céphalique en vue dorsale. 46 : capsule céphalique vue de profil.

FIg. 47 à 49 . - Larve de Micrasema difficile. 47 : capsule céphalique en vue dorsale. 48 : capsule céphalique vue de profil. 49 : tête et plaques thoraciques. 


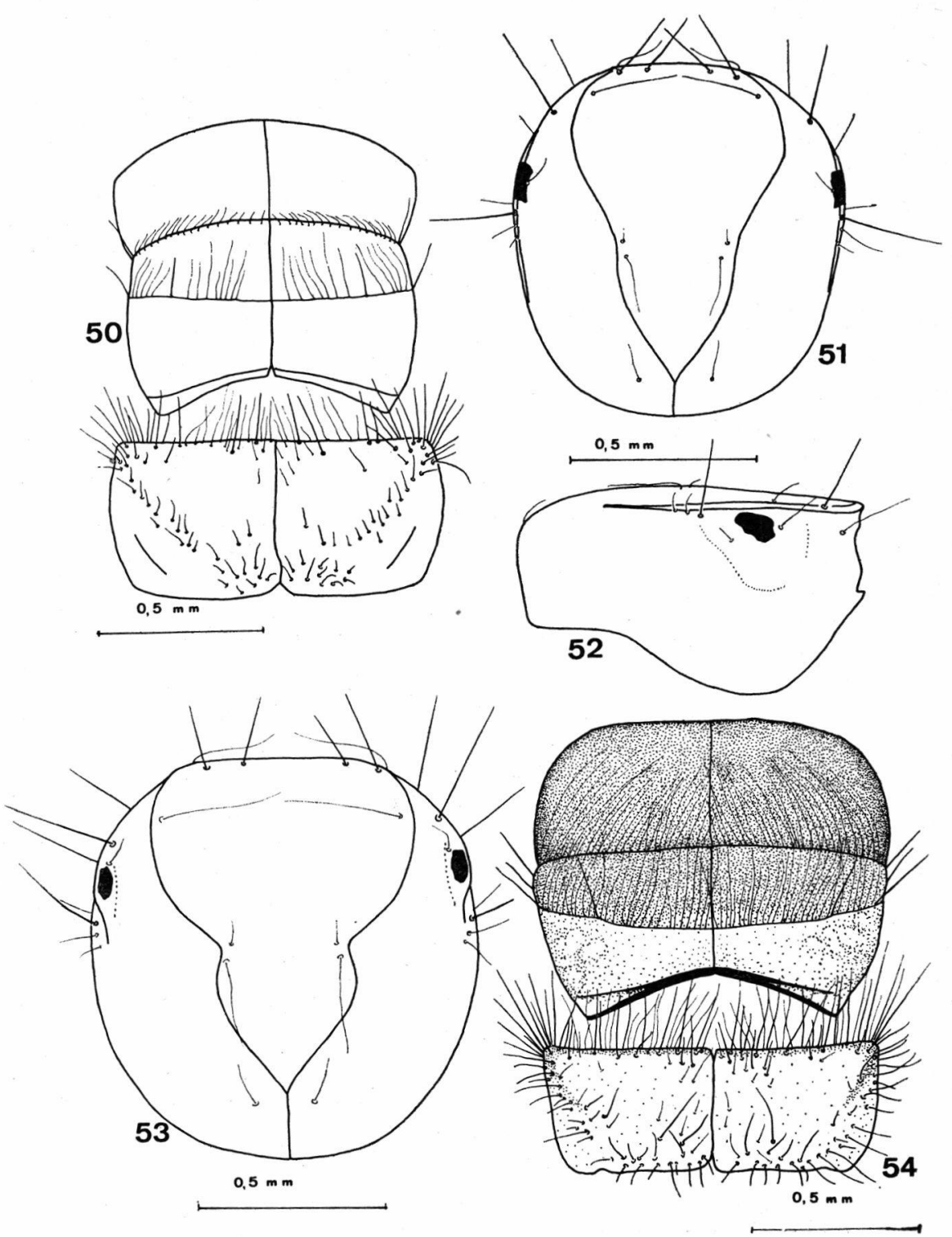

FIg. 50 à 52. $\rightarrow$ Larve de Micrasema cinereum. 50 : tête et plaques du pro. et mésonotum. 51 : capsule céphalique en vue dorsale. 52 : capsule céphalique vue de profil.

Fig. 53 et 54. - Larve de Micrasema togatum. 53 : capsule céphalique en vue dorsale. 54 : tête et plaques du pro. et mésonotum. 


\section{4. - CLE DE DETERMINATION DES ESPECES FRANÇAISES}

1. Fémurs des pattes médianes et postérieures environ deux fois plus longs que les tibias (fig. 14, 15, 16). Tibias médians et postérieurs prolongés ventralement par un processus distal (fig. 14,

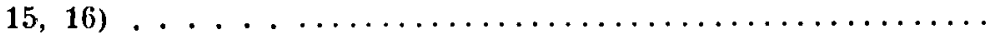

- Fémurs des pattes médianes et postérieures environ aussi longs que les tibias ( $f g .13$ ). Tibias médians et postérieurs non prolongés ventralement par un processus distal (fig. 13) $\ldots \ldots \ldots$

2. Capsule céphalique anguleuse vers l'arrière en vue latérale (fig. 19). Plaques centrales du mesonotum limitées en oblique vers l'arrière ( $f g$. . 21). Etui circulaire en section transversale, composé de particules minérales $(f i g .7) \ldots \ldots \ldots \ldots \ldots \ldots$ $\ldots \ldots \ldots \ldots \ldots \ldots \ldots \ldots$ Oligoplectrum maculatum (Four.) Capsule céphalique arrondie vers l'arrière en vue latérale (fig. 20). Plaques centrales du mésonotum rectangulairés et présentant une limite droite vers l'arrière $(f i g .22,23)$. Etui quadrangulaire en section transversale, composé de matériel végétal disposé perpendiculairement à l'axe $(f i g .8,9) \ldots \ldots \ldots \ldots$

3. Partie proximale des hanches munie de saillies spiniformes sur les pattes médianes et postérieures ( $f g .15)$. Capsule céphalique brun-jaunâtre avec des plages sombres bien délimitées sur le frontoclypeus et sur les pleures (fig. 18). Etui quadrangulaire à bords légèrement arrondis $(f i g .8) \ldots \ldots \ldots \ldots \ldots \ldots$

Brachycentrus subnubilus Curt.

- Partie proximale des hanches sans saillies spiniformes sur les pattes médianes et postérieures (fig. 14). Capsule céphalique brun-châtain, uniformément foncée (fig. 17). Etui quadrangulaire à bords droits (fig. 9) .......... Brachycentrus montanus Klap.

4. Mesonotum composé de quatre plaques chitineuses (fig. 26, 28,

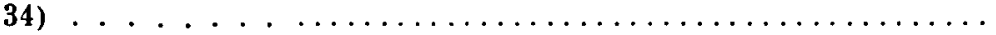

- Mesonotum composé de deux plaques chitineuses (fig. 38, 44,

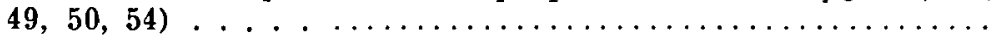

5. Etui minéral (fig. 6) à ouverture postérieure circulaire (fig. 27). Capsule céphalique à bord dorsal aplati en vue latérale (fig. 25). Parties chitineuses de couleur brun-foncé

- Etui minéral ou secrété à ouverture postérieure limitée par 3 à 5 pointes radiales (fig. 32). Capsule céphalique à bord dorsal légèrement arrondi en vue latérale (fig. 30, 33). Parties chiti-

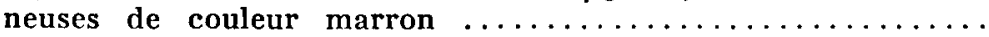

6. Etui composé de fines particules minérales ( $f i g$. 5). Capsule céphalique à bords latéraux arrondis (fig. 29). Bords latéraux des plaques centrales du mesonotum rectilignes (fig. 28) ..... ............................ Micrasema moestum (Hag.)

3. D'après Silfvenius [1905], Micrasema setiferum se placerait aux côtés de Micrasema minimum dont elle differrerait par une coloration plus claire et la présence de 3 crochets dorsaux sur les griffes anales (au lieu de 2 chez M. minimum). 
- Etui entièrement secrété (fig. 12). Capsule céphalique à bords latéraux convergeant vers l'arrière à partir des yeux (fig. 31). Bords latéraux des plaques centrales du mésonotum sinueux (fig. 34) .................. Micrasema morosum (McI..)

7. Tubercules coniques sur les tarses médians et postérieurs ( $f i g .40$, 41). Etui entièrement secrété et légèrement arqué vers l'arrière

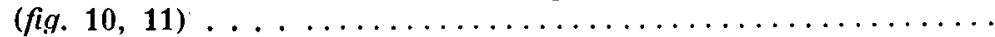

- Pas de tubercule conique sur les tarses médians et postérieurs (fig. 42). Etui en partie secrété, en partie minéral (fig. 2), ou composé de fragments végétaux $(f i g .3,4) \ldots \ldots \ldots \ldots \ldots \ldots$

8. Taches oculaires sur des protubérances prolongées et déprimées vers l'arrière (fig. 36). Plaques mésonotales à bords latéraux se prolongeant vers l'avant (fig. 38). Espèce d'Europe continentale ........................... Micrasema longulum McL.

- Pas de protubérances oculaires (fig. 51). Plaques mésonotales à bords latéraux non prolongés vers l'avant (fig. 50). Espèce corse ....................... Micrasema cinereum Mos.

9. Etui en partie secrété, en partie minéral (fig. 2). Capsule céphalique à bord dorsal aplati ou concave en vue latérale (fig. 48). Plaques mésonotales quadrangulaires (fig. 49). Parties chitineuses de couleur brun-foncé ............... Micrasema difficile Mos.

- Etui composé de fragments végétaux $(f i g, 3,4)$. Capsule céphalique à bord dorsal arrondi en vue latérale (fig. 46). Taches oculaires sur des protubérances (fig. 45, 53). Plaques mésonotales rectangulaires (fig. 44, 54). Parties chitineuses marron ..

10. Espèce d'Europe continentale ........ Micrasema tristellum McL.

— Espèce corse ................ Micrasema togatum (Hag.)

\section{5. - THE FRENCH BRACHYCENTRIDAE LARVAE - KEY}

1. Femurs of meso- and metathoracic legs about twice the length of the tibia (Fig. 14, 15, 16). Tibia of meso- and metathoracic legs with a ventral prolongation (Fig. 14, 15, 16) $\ldots \ldots \ldots \ldots$

- Femurs of meso- and metathoracic legs about the same length as the tibia (Fig. 13). Tibia of meso- and metathoracic legs without a ventral prolongation (Fig. 13) $\ldots \ldots \ldots \ldots \ldots \ldots$

2. Head capsule with the posterior part angular in profile (Fig. 19). Median sclerites on mesonotum obliquely limited towards the posterior (Fig. 21). Larval case with a circular cross-section, made of mineral particles (Fig. 7) $\ldots \ldots \ldots \ldots \ldots \ldots \ldots \ldots$ ..................... Oligoplectrum maculatum (Four.)

- Head capsule with the posterior part curved in profile (Fig. 20). Median sclerites on mesonotum rectangular and not obliquely limited posteriorly (Fig. 22, 23). Larval case with a quadrangular cross section, made of vegetable pieces transversely arranged

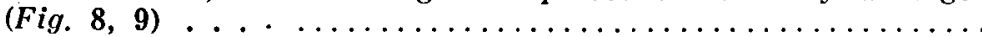

3. Coxae of meso- and metathoracic legs with thorns on proximal part (Fig. 15). A yellowish head capsule with dark patches clearly marked on the frontoclypeus and pleura (Fig. 18). Case 
quadrangular with slightly curved edges (Fig. 8) $\ldots \ldots \ldots$

Brachycentrus subnubilus Curt.

Coxae of meso- and metathoracic legs without thorns on proximal part (Fig. 14). Head capsule uniformly brownish (Fig, 17). Case quadrangular with acute edges (Fig. 9) $\ldots \ldots \ldots \ldots \ldots \ldots$ ......................... Brachycentrus montanus Klap.

4. Mesonotum with 4 dorsal sclerites (Fig. 26, 28, 34) $\ldots \ldots \ldots$ 5

- Mesonotum with 2 dorsal sclerites (Fig. 38, 44, 49, 50, 54) $\ldots .7$

5. Case made of mineral particles (Fig. 6) with the posterior opening circular (Fig. 27). Head capsule dorsally flattened when viewed in profile (Fig. 25). Chitinous parts dark-brown .......

Micrasema minimum 4 McL.

- Case made of mineral particles or secreted by the larva, with the posterior opening limited by 3 to 5 radial points (Fig. 32). Head capsule with the dorsal edge slightly rounded when viewed in profile (Fig. 25). Chitinous parts dark-brown .....

6. Case made of mineral particles (Fig. 5). Head capsule with lateral edges rounded (Fig. 29). Median sclerites on mesonotum with lateral edges straight (Fig. 28) ........................... Micrasema moestum (Hag.)

- Case secreted (Fig. 12). Head capsule with lateral edges converging posteriorly from the eyes (Fig. 31). Median sclerites on mesonotum with lateral edges sinuous (Fig. 34) ...........

Micrasema morosum (McL.)

7. Meso- and Metathoracic tarsi with conical tubercles (Fig. 40, 41). Case entirely secreted and slightly arched posteriorly (Fig. 10,

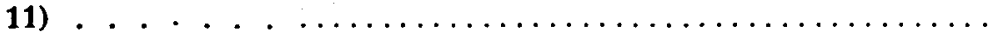

- Meso- and Metathoracic tarsi without conical tubercles (Fig. 42). Case partly secreted, partly made of mineral particles (Fig. 2), or made of vegetable fragments $(\mathrm{Fig} .3,4) \ldots \ldots \ldots \ldots \ldots \ldots$

8. Eyes located on raised areas which are extended and tapered towards the posterior (Fig. 36). Mesonotal plates with lateral edges produced anteriorly (Fig. 38). Continental species ......

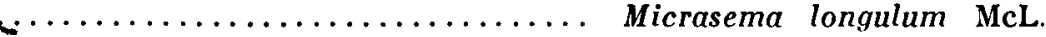

- Eyes not located on raised areas (Fig. 51). Mesonotal plates with lateral edges not produced anteriorly. (Fig. 50). Corsican species ...................... Micrasema cinereum Mos.

9. Case partly secreted, partly made' of mineral particles (Fig. 2). Head capsule with a very flattened dorsal edge when viewed in profile (Fig. 48). Mesonotal plates quadrangular (Fig. 49). Chitinous parts dark brown ........... Micrasema difficile Mos.

- Case made of vegetable fragments (Fig. 3, 4). Head capsule with a curved dorsal edge when viewed in profile (Fig. 46). Eyes on raised areas (Fig. 45, 53). Mesonotal plates rectangular

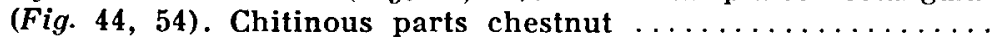

10. Continental species .............. Micrasema tristellum McL. - Corsican species .............. Micrasema togatum (Hag.)

4. From Silfvenius [1905] Micrasema setiferum would be close to Micrasema minimum, the difference being a lighter coloration and 3 dorsal spines on the anal claws (instead of 2 for Micrasema minimum). 


\section{6. - DIE FRANZÖSISCHEN BRACHYCENTRIDEN LARVEN - BESTIMMUNGSTABELLE}

1. Femora der Mittel- und Hinterbeine ungefähr zweimal länger als die Tibien (Fig. 14, 15, 16). Mittel- und Hintertibien auf der Ventralseite mit einem distalen Dorn versehen (Fig. 14, 15, 16)

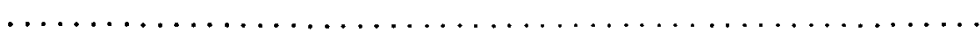

- Femora der Mitte- und Hinterbeine ungefähr ebenso lang wie die Tibien (Fig. 13). Mittel- und Hintertibien ohne distalen Dorn

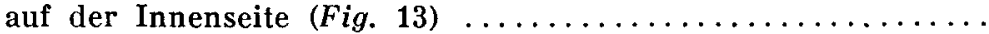

2. Kopfkapsel in Seitenansicht nach hinten eckig (Fig. 19). Mesosklerite des Mesonotums nach hinten schräg abgegrenzt (Fig. 21). Köcher mit rundem Querschnitt, aus Sandkörnchen zusammengesetzt (Fig. 7) ..... Oligoplectrum maculatum (Four.)

- Kopfkapsel in Seitenansicht nach hinten gerundet (Fig. 20). Mesosklerite des Mesonotums rechteckig und nach hinten gerade abgegrenzt (Fig. 22, 23). Köcher mit viereckigem Querschnitt, bestehend aus Pflanzen-material, das senkrecht zur Längsachse

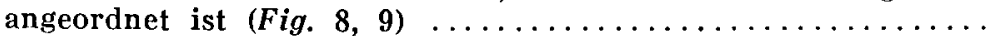

3. Hüften der Mittel- und Hinterbeine proximal mit Dornen besetzt (Fig. 15). Kopfkapsel gelblich-braun mit scharf begrenzten dunklen Flecken auf dem Frontoclypeus und den Pleuren (Fig. 18). Köcher viereckig mit leicht gerundeten Kanten (Fig. 8) .................. Brachycentrus subnubilus Curt.

- Hüften der Mittel- und Hinterbeine ohne Dorne (Fig. 14). Kopfkapsel rotbraun, einheitlich dunkel (Fig. 17). Köcher von quadratischem Querschnitt mit geraden Kanten (Fig. 9) ......... ....................... Brachycentrus montanus Klap.

4. Mesonotum auf der Dorsalseite mit 4 Sikleriten (Fig. 26, 28, 34). 5

- Mesonotum auf der Dorsalseite mit 2 Skleriten (Fig. 38, 44, 49,

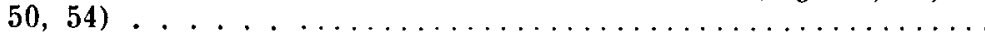

5. Köcher aus Sandkörnchen (Fig. 6) mit runder Hinteröffnung (Fig. 27). Dorsaler Rand der Kopkapsel in Seitenansicht abgeflacht (Fig. 25). Die Chitinhaltigen Partien dunkelbraun gefärbt $\ldots \ldots \ldots \ldots \ldots \ldots \ldots \ldots \ldots \ldots$ Micrasema minimum ${ }^{5}$ McL.

- Köcher aus Sandkörnchen oder glatt mit 3 oder 5 radialen Spitzen als Begrenzung der Hinteröffnung (Fig. 32). Dorsaler Rand der Kopfkapsel in Seitenansicht leicht gerundet (Fig. 30, 33). Die chitinhaltigen Partien kastanienbraun gefärbt .......

6. Köcher aus feinen Sandkörnchen zusammengesetzt (Fig. 5). Kopfkapsel kugelig mit gerundeten Seitenrändern (Fig. 29). Seitenränder der Zentralsklerite des Mesonotums geradlinig (Fig. 28) .................. Micrasema moestum (Hag.)

5. Nach Srrfvenius [1905] stände Micrasema setiferum nahe bei Micrasema minimum von der Sie sich jedoch durch eine hellere Färbung und des Vorhandensein von 3 Dorsalhacken an den Analfüssen (an Stelle von 2 bei Micrasema minimum) unterscheidet. 
- Köcher ganz glatt (Fig. 12), Seitenränder der Kopfkapsel von den Augen ab nach hinten zusammenlaufend (Fig. 31). Seitenränder der Zentralsklerite des Mesonotums wellig (Fig. 34) ...

Micrasema morosum (McL.)

7. Konische Höcker auf den Mittel- und Hintertarsen (Fig. 40, 41). Höcher ganz glatt und leicht nach hinten gebogen (Fig. 10, 11).

- Keine konischen Höcker auf Mittel- und Hintertarsen (Fig. 42). Köcher teilweise glatt, teilweise aus Sandkörnchen (Fig. 2) oder Pflanzenbruchstücken zusammengesetzt (Fig. 3, 4) ........

8. Augenflecken auf Protuberanzen, nach hinten verlängert und eine Grube bildend (Fig. 36). Mesonotal Sklerite deren Seitenränder ordwärts auslaufen (Fig. 38). Art aus Kontinentaleuropa ........................ Micrasema longulum McL.

- Keine Protuberanzen der Augenzone (Fig. 51). Mesonotalsklerite ohne nach vorne verlängerte Seitenränder (Fig. 50). Art aus Korsika ..................... Micrasema cinereum Mos.

9. Köcher teilweise glatt, teilweise aus Sandkörnchen zusammengesetzt (Fig. 2). Dorsalrand der Kopfkapsel in Seitenansicht stark abgeflacht (Fig. 48). Mesonotalsklerite quadratish (Fig. 49). Chitinhaltige Partien dunkelbraum gefärbt .. Micrasema di:ficile Mos.

- Köcher aus Pflanzenbruchstücken zusammengesetzt (Fig. 3, 4). Dorsalrand der Kopfikapsel in Seitenansicht gerundet (Fig. 46). Augenflecken auf Protuberanzen (Fig. 45, 53). Mesonotalsklerite rechteckig (Fig. 44, 54). Chitinhaltige Partien Kastanienbraun.

10. Art aus Kontinentaleuropa ........ Micrasema tristellum McL. - Art aus Korsika ............... Micrasema togatum (Hag.)

\section{TRAVAUX GITES}

Baudorn (J.). 1968. - Inventaire des Trichoptères de Basse-Auvergne. Observations écologiques et biologiques. Annls St. biol. Besse-enChandesse, 3 : 1-40.

Berland (L.) et Mosely (M. E.). 1936. - Catalogue des Trichoptères de France, tome I. Annls Soc. ent. Fr., 105 : 111-144.

Botosaneanu (L.). 1967. - Trichoptera in Illies J. Limnofauna Europaea. Stuttgart : 285-309.

Décamps (H.). 1967 a. - Introduction à l'étude des Trichoptères des Pyrénées. Annls Limnol., 3 (1) : 101-176.

Décamps (H.). 1967 b. - Ecologie des Trichoptères de la vallée d'Aure (Hautes-Pyrénées). Annls Limnol., 3 (3) : 399-577.

Décamps (H.). 1968. - Vicariances écologiques chez les Trichoptères des Pyrénées. Annls Limnol., 4 (1) : 1-50.

Giudiceldi: (J.). 1968. - Recherches sur le peuplement, l'écologie et la biogéographie d'un réseau hydrographique de la Corse centrale. (Thèse Sciences, Aix-Marseille, C.N.R.S., A.O. 2478), 437 p.

Hickin (N. E.). 1943. - Larvae of the British Trichoptera. 17. Brachycentrus subnubilus Curtis. Proc. $R$. ent. Soc. Lond. (A) 18 : 81-83.

Krapalek (F.). 1893. - Untersuchungen über die Fauna der Gewässer Böhmens. I. Metamorphose der Trichopteren, II, Serie. Arch. Naturw. Land Durchforsch. Böhm., 8 (6) : 1-142. 
Krapalek (F.). 1917. Uber die von Herrn Prof. A. Hetschko in Korsica gesammelten Neuropteroiden nebst Bemerkungen über einige ungenügend bekannte Arten. Wien ent. Zeit., 36 (6/8) : 193-208.

LePneva (S. G.). 1966. - La faune de l'U.R.S.S., Trichoptera, 2, 522 p. Moscou (en russe).

Lestage (S. A.). 1921. - Trichoptera in Rousseau (E.) : Les larves et nymphes aquatiques des insectes d'Europe. 967 p. Bruxelles.

MAC LACHLaN (R.). 1874-1884, - A monographic revision and synopsis of the Trichoptera of the european fauna. 523 p., pl. 1-51; suppl. I et II : 1-103, pl. 52-59. First Additional Suppl., 76 p., 7 pl. London.

Maillet (P.) et Carasso (N.). 1955. - Catalogue systématique des larves des Trichoptères récoltés dans la région des Eyzies (Dordogne France). Espèces à fourreau. Hydrobiologia, 7 (4) : 387-393.

Mosely (M. E.). 1934. - New Trichoptera in the French Pyrenees. Ann. Mag. nat. Hist., 10 (13) : 433-444.

Nielsen (A.). 1943. - Postembryonale Entwicklung und Biologie der rheophilen Köcherfliege Oligoplectrum maculatum Fourcroy. $K$. danske Vidensk. Selsk. Mddr., 19 (2) : 1-87 + 3 pl.

Silfvenius (A.). 1905. - Beiträge zur Metamorphose der Trichopteren. Acla Soc. Fauna Flora Fenn., 27 (6) : 1-168.

Thieneman (A.). 1905. - Biologie der Trichopterenpuppen. Zool. Jarhb., $22(5): 489-574$.

ULMER (G.). 1903. - Beiträge zur Metamorphose der deutschen Trichopteren. Allg. Z. Ent. : 309-311.

ULMER (G.). 1909. - Trichoptera, in BRAUER: Die Süsswasserfauna Deutschlands. Jena, 5-6 : 1-326.

Wiggins (G. B.). - Additions and revisions to the Genera of North American Caddisflies of the Family Brachycentridae with special reference to the larval stages (Trichoptera), Can. Ent., 97:10891106.

Laboratoire d'Hydrobiologie

(Equipe de Recherche Associée au C.N.R.S.), 118, route de Narbonne, 31 -Toulouse. 